\title{
Evaluating the Impact of Beacon Interval and Neighbor Timeout Timer on the Performance of Geographical Routing in FANETs
}

\author{
Vikramjit Singh $^{1,{ }^{*}}$ Krishna Pal Sharma $^{1,}$ Harsh K Verma ${ }^{1}$ \\ ${ }^{1}$ Department of Computer Science and Engineering, Dr B R Ambedkar National Institute of Technology, Jalandhar \\ 144011, Punjab, India \\ 'Corresponding author. Email: bhathal2017@gmail.com
}

\begin{abstract}
Geographical routing provides a robust and scalable routing solution for Flying Ad Hoc Networks (FANETs). In this, the reliability of forwarding decisions depends on the accuracy of the position information of neighboring nodes stored in the neighbor table. Each node broadcasts beacon packets to exchange its position with neighboring nodes after a fixed time interval known as beacon interval (BI) to construct the neighbor table. The node that receives the beacon packet adds an entry in its neighbor table for the originator of a beacon. That entry is valid only for a specific time called neighbor timeout timer $\left(\mathrm{N}_{\mathrm{TOT}}\right)$. The fixed value setting of beacon interval and neighbor timeout timer may deteriorate the routing performance in a dynamic scenario like FANETs, where network characteristics change frequently. Therefore, in this paper, we investigate the impact of fixed beacon interval and fixed neighbor timeout timer on the performance of geographical routing. To do so, extensive simulations are conducted by varying the value of beacon interval and neighbor timeout timer for different network settings in ns-3. The simulation results demonstrate that the beacon interval and neighbour timeout timer significantly affects the performance of geographical routing. Also, geographical routing exhibits the best performance at $\mathrm{BI}=0.5 \mathrm{~s}$ and $\mathrm{N}_{\mathrm{TOT}}=1.5 \mathrm{~s}$ in most network conditions.
\end{abstract}

Keywords: Beacon interval, FANETs, Geographical routing, Neighbor timeout timer, Unmanned Aerial Vehicle $(U A V)$.

\section{INTRODUCTION}

The recent technological advancements have paved the way for the development of small-sized unmanned aerial vehicles (UAVs), which are helpful in a wide range of military and civil applications. Equipped with various sensors and communication capabilities, UAVs can perform tasks in a cooperative fashion, giving rise to a new networking paradigm known as flying ad hoc networks (FANETs) [1], [2]. However, due to unique features of small-sized UAVs (like high mobility, sparse deployment and energy constraints) the design of efficient routing protocol is a challenging task. Various topology-based routing schemes have been proposed to overcome these challenges [3]-[5]. The issue related to topology-based routing is that nodes need to construct an entire route before sending data packets, resulting in high control overhead due to the dynamic topology of FANETs. In such scenarios, geographical routing is considered a promising approach that provides simple and scalable routing solutions [6], [7]. In this, nodes only need to maintain one-hop neighbor information for routing decisions and incur low control overhead, making it suitable for dynamic networks. The accuracy of neighborhood information affects the reliability of routing decisions to a large extent and maintaining an accurate view of local topology is a challenging task when nodes are moving at high speed. In this regard, one of the widely used solutions is periodic beaconing, in which each UAV transmits beacons or hello packets at fixed intervals to announce its position. All neighboring UAVs make an entry in their neighbor table for the beacon's transmitter. This entry is valid for a fixed time called neighbor timeout timer $\left(\mathrm{N}_{\mathrm{ToT}}\right)$ and is removed after its expiration.

The first issue with periodic beaconing is that UAVs need to transmit beacons at a high rate to maintain the 
exact view of network topology, resulting in higher control overhead and energy consumption. On the other hand, the increase in beacon interval (BI) (means beacons transmitted at a lower rate) leads to outdated or stale entries in the neighbor table. Thus there is a tradeoff between the accuracy of neighborhood information in the neighbor table and control overhead. Another issue is assigning the same neighbor timeout timer for all neighboring nodes irrespective of their position, speed and direction of motion. There is a high probability of link breakage much before the $\mathrm{N}_{\text {ToT }}$ with neighboring nodes present at the border of transmission range and moving in the opposite direction.

This paper aims to analyze the impact of beaconing and neighbor timeout timer on the performance of geographic routing in FANETs. To accomplish this, extensive simulations are performed for different $\mathrm{BI}$ and $\mathrm{N}_{\text {To T }}$ by varying the node density, traffic flows and node speed.

\section{BACKGROUND AND RELATED WORK}

Generally, in geographical routing, greedy forwarding is used to forward the data packets from source to destination. In this, the forwarding node selects the next-hop closest to the destination from its neighbor list. To do this, each node requires location information of the destination and neighboring nodes. The location of destination is assumed to be known in advance (in case of fixed destination) or can be obtained by using the location services [8]. The position information of neighboring nodes is achieved by exchanging beacons at regular intervals. In greedy perimeter stateless routing (GPSR) [9], the BI is fixed at $1 \mathrm{~s}$ and $\mathrm{N}_{\text {ToT }}$ is calculated as:

$\mathrm{N}_{\mathrm{ToT}}=\alpha^{*} \mathrm{BI}$

where the value of $\alpha$ is $4.5 \mathrm{~s}$

Several works have been done by researchers for mobile ad hoc networks (MANETs) and vehicular ad hoc networks (VANETs) to control the beacon overhead and to maintain the updated list of neighboring nodes. The impact of location errors caused by mobility and beacon interval on the performance of geographical routing in MANET is analyzed in [10], [11]. In [12], the limitations of periodic beaconing are explored, and authors suggested various dynamic beaconing schemes to mitigate them. In [13], dynamic adjustment of beacon interval is proposed by using the link change rate. In [14], each node utilizes a fuzzy logic controller to reduce the beacon overhead by considering node speed and the number of neighboring nodes. The authors in [15] evaluated the effect of neighbourhood discovery time on the performance of geographic routing in mobile sensor network and calculated the suitable time interval for neighbourhood discovery after considering the communication range, speed and number of neighboring nodes. In [16], a beaconing scheme is proposed to maintain the trade-off between the accuracy of the neighbor table and beacon overhead. The authors in [17] and [18] exploited vehicles' mobility characteristics to design adaptive beaconing schemes for VANETs. In [19], the authors proposed the adaptation scheme to adjust the beacon frequency and beacon transmission power to control the congestion due to beacon messages. However, for FANETs, few proposals are available to overcome the adverse effects of periodic beaconing. For instance, in [20], the adaptive hellointerval scheme is proposed for FANETs to reduce energy consumption while improving network performance. Additionally, in [21], an adaptive beacon scheme is presented in which the fuzzy control system is used to adjust the frequency of beacons dynamically. In [22], a predictive mathematical model is used to adapt the beacon interval of ad hoc on-demand distance vector (AODV) protocol. In this, beacon rate is controlled by considering the dimensions of mission area, velocity and transmission range of UAVs. In [23], mobility information of UAV is exploited to adjust the beacon transmission rate to improve the accuracy of position information stored in neighbour table.

To the best of author's knowledge, no such study has been found that analyzed the impact of beacon intervals for geographical routing in FANETs. As the characteristics of FANET is different from MANETs and VANETs (in terms of mobility, topology change, speed and propagation loss), the studies done for these networks may not be used directly and need to be analyzed for FANETs [24]. Therefore, in this work, the impact of beacon interval on the performance of geographical routing in FANET is analyzed by varying the node speed, node density and number of traffic flows. Moreover, the effect of assigning fixed $\mathrm{N}_{\text {ToT }}$ to all neighbor table entries is also explored.

\section{IMPACT OF BEACON INTERVAL AND NEIGHBOR TIMEOUT TIMER}

In this section, the impact of beacon interval and neighbour timeout timer on the performance of geographic routing is discussed.

\subsection{Impact of beacon interval}

In geographical routing, beacons are broadcasted by nodes at regular interval to exchange their position information with neighboring nodes. In highly mobile scenario, where position of nodes changes frequently, nodes need to broadcast beacons at higher rate to maintain updated topological information in neighbor table. However, the transmission of beacons at higher rate not only consumes extra bandwidth and energy but also interferes with the transmission of data packets 
which increases the chances of collision and congestion. On the contrary, larger beacon interval leads to outdated and inaccurate position information in the neighbor table, resulting in non-optimal routing decisions. As shown in Figure 1, three UAVs $U_{i}, U_{j}$ and $U_{k}$ are flying with velocity $V_{i}, V_{j}$ and $V_{k}$ respectively and exchange their position information at BI interval. At time $t_{1}$, UAVs exchange beacons among themselves and position information of neighboring nodes are stored in their respective neighbor tables. At time $t+\Delta t, U_{\text {i gets a }}$ packet for destination $(d)$ and searches its neighbor table for optimal next hop closest to the destination according to greedy forwarding. $U_{i}$ selects $U_{\mathrm{j}}$ as next hop since it is closest to $d$ as per neighbor table but in reality, the position of nodes has changed since the last transmission of beacons and currently, $U_{k}$ is the closest

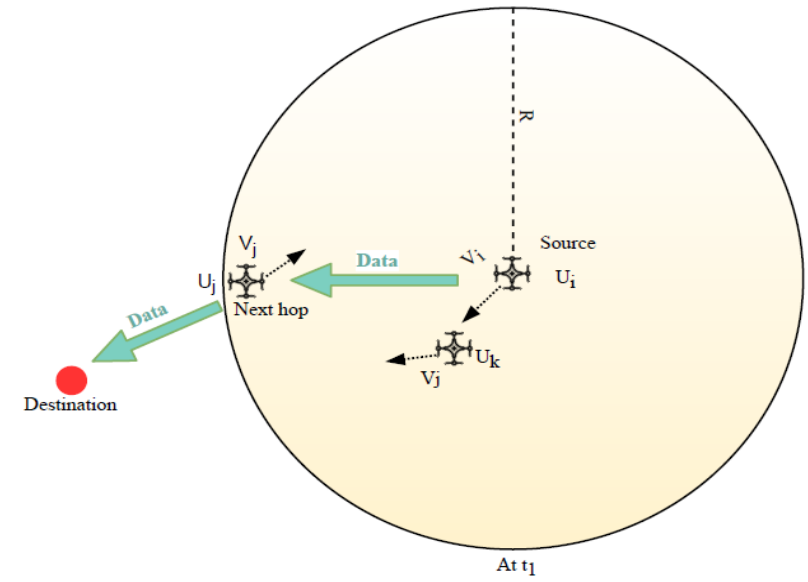

Figure 1 Non-optimal routing decision

Therefore, we try to figure out experimentally that how the transmission rate of beacons influences the performance of geographical routing in different network settings. For that, simulations are conducted for different values of BI (ranging from $0.25 \mathrm{~s}$ to $3 \mathrm{~s}$ ) by varying the node density, speed and traffic flows.

\subsection{Impact of neighbor timeout timer}

When node receives a beacon from its neighboring nodes then it extracts the position information and store in its neighbor table. This information is valid for particular time interval known as neighbor timeout timer. If node's neighbor table already contains the entry of its neighboring node, then beacon information is used for updating the position information and timeout timer. When a node needs to forward a packet, it takes routing decisions based on the information present in the neighbor table. In this way, the accuracy of information present in neighbor table affects the effectiveness of routing decisions to a great extent. The accuracy of the neighbor table can be improved by exchanging the beacons at a high rate. However, there are several drawbacks related to the frequent transmission of beacon packets. Moreover, the accuracy of neighbor next hop to $d$. $U_{i}$ takes sub-optimal routing decision for some time, and after BI time neighbor tables of all nodes get updated with the current status of one-hop nodes only if we assume beacons are not lost during transmission. So in best case, $U_{i}$ makes routing decision sub-optimally for the following time $(\mathrm{T})$.

$\mathrm{T}=\mathrm{BI}-\Delta \mathrm{t}$

Here, the time for which sub-optimal decisions are taken is dependent on $\mathrm{BI}$ and $\Delta t$. The estimation of $\Delta t$ is not possible in real-time scenario, but it is evident that as the BI increases, the time for non-optimal routing decisions increases due to stale position information in the neighbor table.

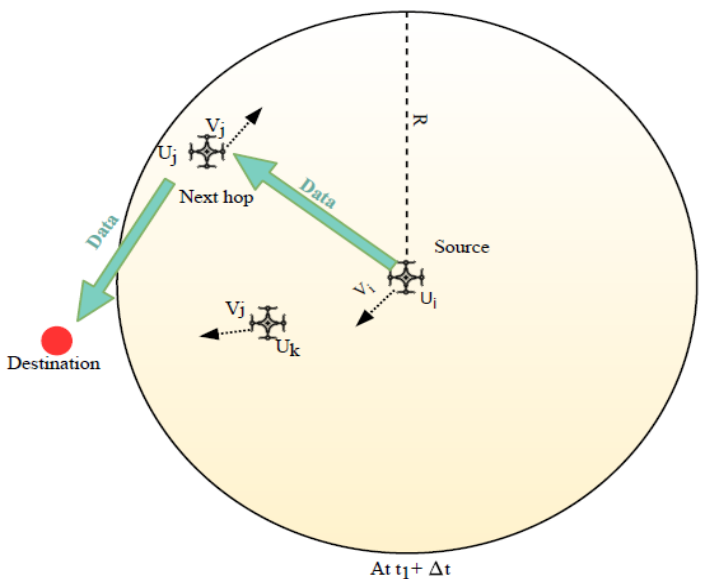

table not only depends upon the beacon interval but also on the time for which the entry of neighboring node is stored in neighbor table. The information present in neighbor table becomes stale after some time due to the mobility of nodes. Such neighbor table entries are also termed as false neighbors [25], and their presence gives rise to a problem known as the lost link problem [10]. For instance, as shown in Figure 2, UAVs $U_{i}$ and $U_{j}$ exchange beacons at time $t_{1}$ and stored position information for $\alpha^{*} B I$ time in their neighbor table. At $t_{1}+\Delta t, U_{j}$ leaves the communication range of $U_{i}$, but its entry is already present in neighbor table of $U_{j}$. Now, $U_{i}$ selects $U_{j}$ as the next hop for each packet which is for destination $(d)$. This situation remains there for a time (T).

$\mathrm{T}=\mathrm{N}_{\mathrm{ToT}}-\Delta \mathrm{t}$

$\mathrm{T}=\alpha^{*} \mathrm{BI}-\Delta \mathrm{t}$

Here, $\alpha$ is constant whose value is 2 in most cases. There is a trade-off, as a very large value of $\alpha$ leads to stale entries, and a short value leads to early removal of neighbor table entries. Therefore, to evaluate the impact of $\mathrm{N}_{\text {TOT }}$, we conduct simulations for different values of $\alpha$. 

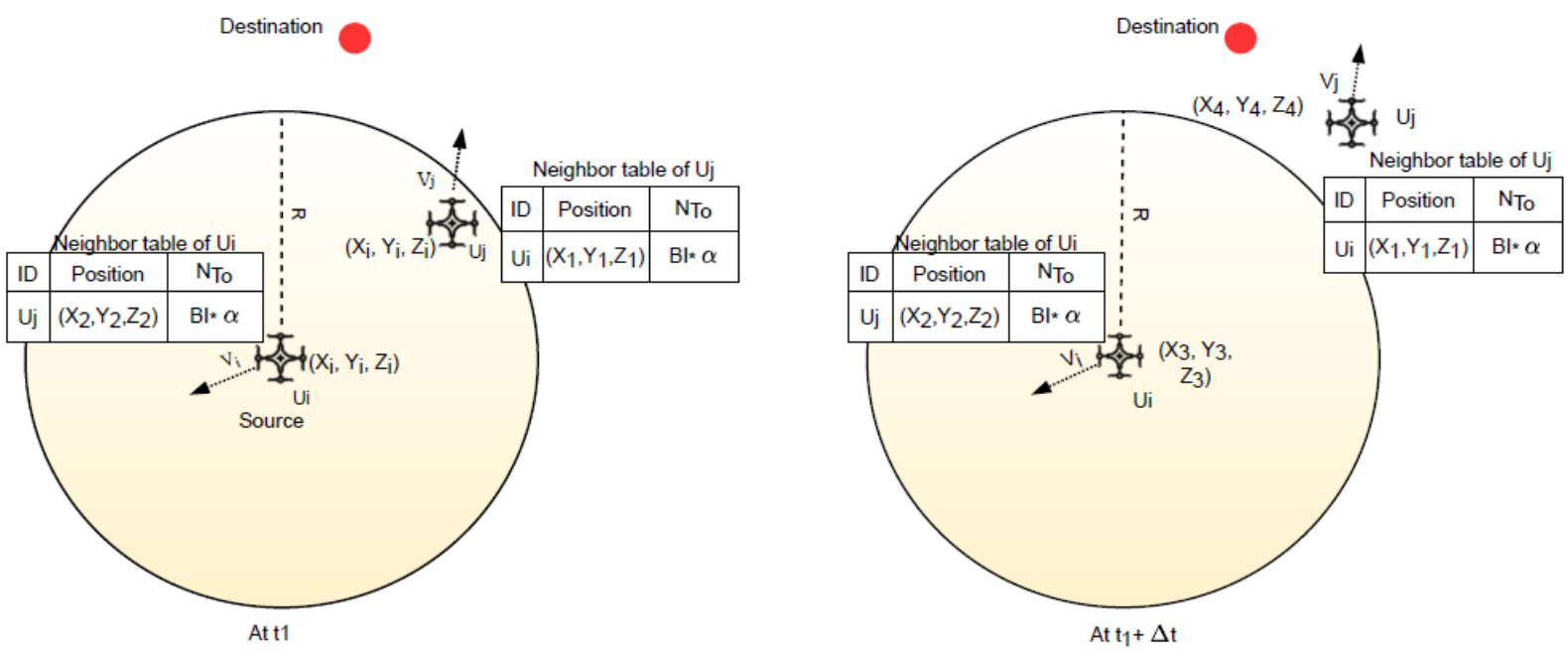

Figure 2 Lost link problem

\section{PERFORMANCE EVALUATION}

In this section, the impact of beacon interval and neighbor timeout timer on the performance of geographical routing in FANETs is analysed experimentally by conducting a set of simulations. For that, GPSR (one of the widely used algorithm for geographical routing) is implemented in ns-3 and simulations are performed by varying $\mathrm{BI}$ and $\mathrm{N}_{\mathrm{ToT}}$. In each simulation, multiple UAVs ranging from 20 to 50 are randomly deployed in a field of size $2000 \mathrm{~m} * 2000$ $\mathrm{m} * 150 \mathrm{~m}$. As a media-access-control (MAC) layer protocol, IEEE $802.11 \mathrm{~b}$ is used, and each UAV flies according to the $3 \mathrm{D}$ version of the Gauss Markov mobility model. The performance metrics considered in this analysis are described as follows:

The performance metrics considered in this analysis are described as follows:

Packet delivery ratio- It is defined as the total number of packets received divided by the total number of packets transmitted.

Mac transmission failures- It represents the total number of transmissions of data packets failed at Mac layer.

\subsection{Effect of beacon interval}

This subsection analyses the performance of geographical routing for different values of BI in terms of packet delivery ratio and mac transmission failures. The value of BI ranges from $0.25 \mathrm{~s}$ to $3 \mathrm{~s}$, and the value of $\mathrm{N}_{\text {TOT }}$ is fixed at $2 * \mathrm{BI}$.

\subsubsection{Packet delivery ratio}

Figure 3 shows the influence of beacon interval on packet delivery ratio for varying numbers of nodes in

the network. It is seen that BI at 1s exhibits a superior delivery ratio for low node density and at $0.5 \mathrm{~s}$ performs better as the number of nodes in the network increases. In addition, the performance of GPSR improves with the decrease in $\mathrm{BI}$ and this trend continues only up to $0.5 \mathrm{~s}$ and after that performance start deceasing.

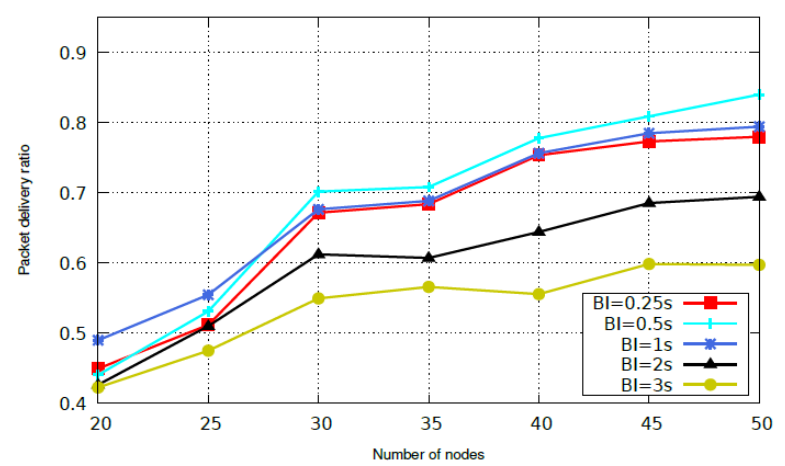

Figure 3 Effect of BI on delivery ratio for varying node density

The accuracy of neighbor table improves as there is decrease in BI since there is frequent exchange of neighborhood information. However, the decrease in BI also increases beacon overhead that leads to congestion and interference with data transmission. Therefore, when $\mathrm{BI}$ is set at $0.25 \mathrm{~s}$ the delivery ratio starts decreasing which is due to the overhead caused by beacons. It is worth mentioning that at $\mathrm{BI}=3 \mathrm{~s}$, GPSR shows underperformance as nodes are unable to maintain the updated neighbour list due to long beacon interval. 


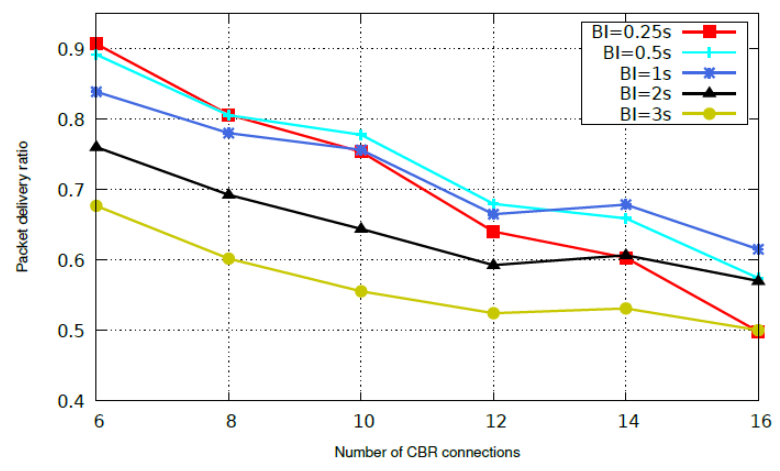

Figure 4 Effect of BI on delivery ratio for different CBR connections

Figure 4 shows the packet delivery ratio versus number of constant bit rate (CBR) flows for different BI. It is interesting to note that GPSR with $\mathrm{BI}=0.25 \mathrm{~s}$ outperforms all other beaconing settings when the traffic flow in network is low. Then its performance starts decreasing as the traffic load in the network increases. This is because at a high beacon rate, the possibility of collisions and network congestion increases when there is an increase in the number of data packets. On the other hand, GPSR with $\mathrm{BI}=3 \mathrm{~s}$ shows the least packet delivery ratio. The reason behind this is that the exchange of location information after every $3 \mathrm{~s}$ in dynamic scenario failed to maintain the updated and accurate neighborhood information. As a result, outdated information in the neighbor table leads to inaccurate and sub-optimal routing decisions.

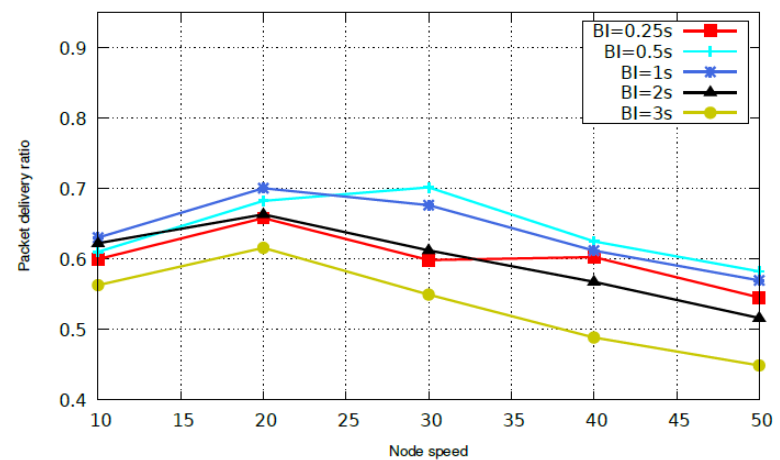

Figure 5 Effect of BI on delivery ratio for varying node speed

Figure 5 shows the packet delivery ratio for different node speeds. The beacon interval at $1 \mathrm{~s}$ outperforms for low mobile scenarios, and $0.5 \mathrm{~s}$ performs better when there is an increase in node speed. At low beacon intervals, nodes can capture accurate topological information, which improves the routing performance. However, when $\mathrm{BI}=0.25 \mathrm{~s}$, the network shows a low packet delivery ratio due to increased beacon overhead. Thus, lowering the beacon interval is favourable up to a certain extent, and after that it starts degrading the network performance.

\subsubsection{Mac transmission failures}

Figure 6 shows the number of mac layer transmission failures of data packets versus the speed of nodes in the network. The reasons behind the transmission failure at Mac layer can be collisions, interference or link failure due to mobility. It is seen that with the increase in node speed and BI, the number of failures increases.

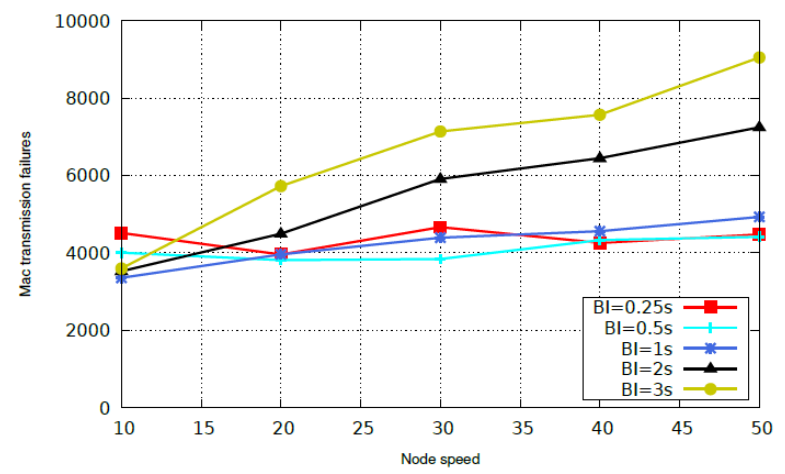

Figure 6 Effect of BI on transmission failures for varying node speed

In GPSR, when a node gets a packet to forward, it selects the most appropriate node (as per rules) from its neighbor table as a next hop. There is a possibility that the selected next-hop may leave the communication range of the forwarding node due to mobility. In that case, transmission failures happen at Mac layer and after multiple re-transmissions packets are dropped by node if acknowledgement is not received. Thus, at higher BI, there is a surge in the proportion of stale entries, leading to erroneous routing decisions and consequently resulting in higher transmission failures at Mac layer.

\subsection{Effect of neighbour timeout timer}

In this subsection, the impact of neighbour timeout timer on routing performance is analyzed. To do so, the value of $\mathrm{BI}$ is fixed at $0.5 \mathrm{~s}$, and the value of $\mathrm{N}_{\mathrm{ToT}}$ is calculated according to $\alpha^{*} \mathrm{BI}$, where $\alpha$ ranges from 1 to 5.

\subsubsection{Packet delivery ratio-}

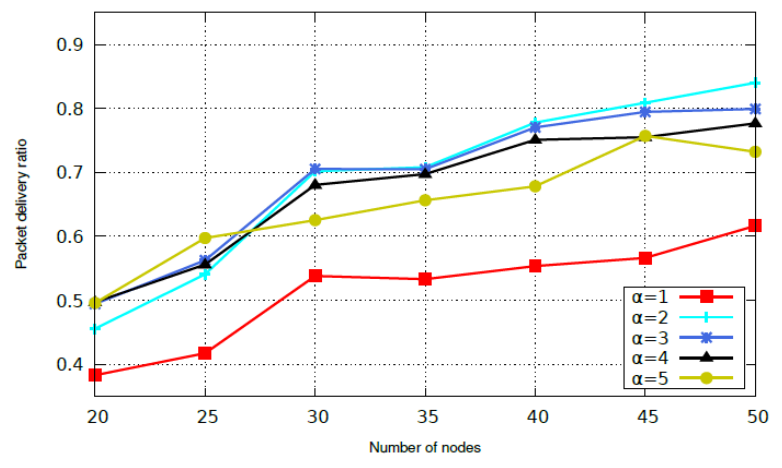

Figure 7 Packet delivery ratio for varying node density 
Figure 7 illustrates the packet delivery ratio for different neighbor timeout timer. In sparse network, GPSR with large $\mathrm{N}_{\text {ToT }}$ performs better, and as the density increases, $\alpha=2$ outperforms other settings. Moreover, the network performance degrades for very low $\mathrm{N}_{\text {ToT. }}$. This is because entries related to neighboring nodes are removed too early, and the neighbor table failed to reflect the exact view of topology, which subsequently affects the routing decisions [26-28].

Figure 8 and Figure 9 shows packet delivery ratio versus the node speed and number of CBR connections, respectively. At $\alpha=2$ and $\alpha=3$ demonstrates the best performance as compared to other settings. The assignment of very low and very high value to $\mathrm{N}_{\text {ToT }}$ negatively affect the routing performance. The low $\mathrm{N}_{\mathrm{ToT}}$ does not reflect exact topology, and large $\mathrm{N}_{\mathrm{ToT}}$ leads to an increase in the number of false neighbors [29].

Figure 10 shows the transmission failures at Mac layer versus the speed of nodes in the network. Here, $\alpha=2$ and $\alpha=3$ outperforms other settings of $\mathrm{N}_{\text {Tот. }}$. The $\alpha=3$ shows superior performance for the low mobility, and as the node speed increases, $\alpha=2$ starts outperforming. This is due to the rise in the number of false entries in neighbor table due to increased node speed. In highly mobile scenario, setting a large neighbor timeout increases the number of false neighbors whose entries are present in the neighbor table of nodes even though they have left their communication range [30-32].

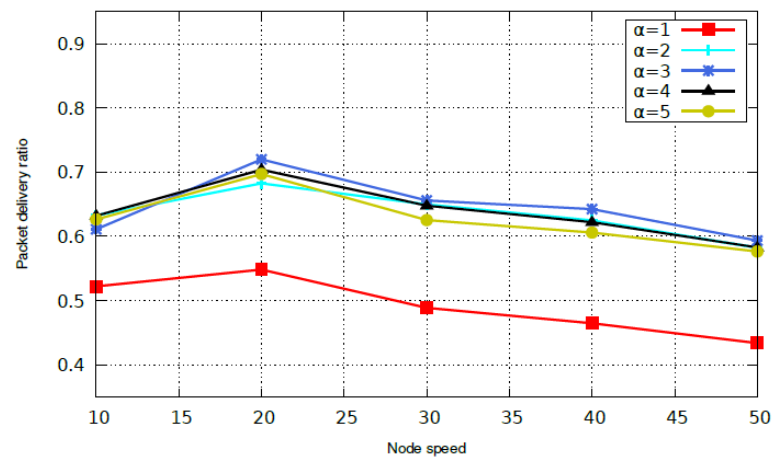

Figure 8 Packet delivery ratio for varying node speed

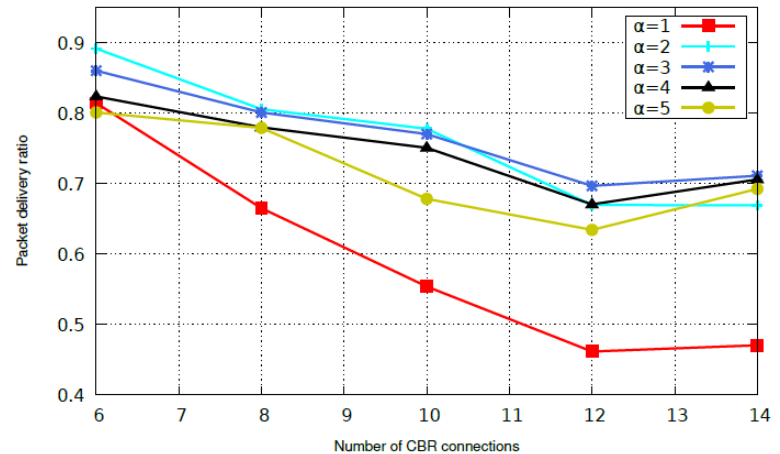

Figure 9 Packet delivery ratio for different CBR connections

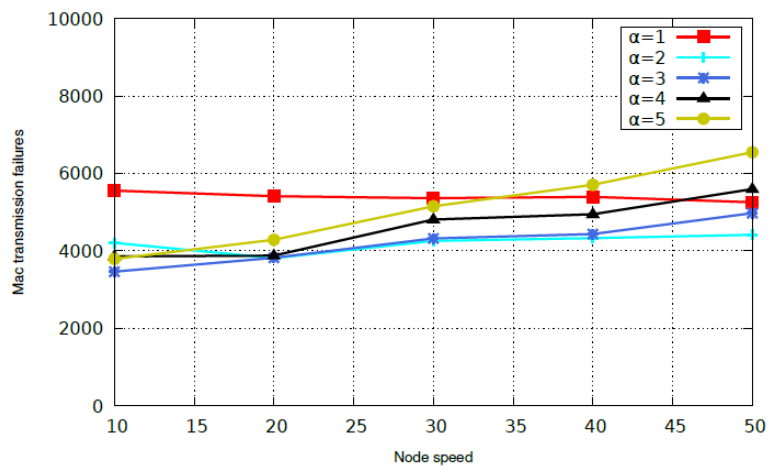

Figure 10 MAC transmission failures for varying node speed

As a result, selecting a false neighbor as a next hop leads to transmission failures at Mac layer [33-39].

\section{CONCLUSION AND FUTURE SCOPE}

In this work, the impact of beacon interval and neighbor timeout timer on the performance of geographical routing is evaluated. For that, multiple sets of simulations are conducted for different beacon intervals and neighbor timeout timers. The simulation results demonstrate that none of the beacon interval and neighbor timeout timer settings can achieve the best performance in all network conditions. $\mathrm{BI}=1 \mathrm{~s}$ exhibit best performance for sparse connectivity and lower mobility. On the other hand, for highly mobile and dense scenarios, $\mathrm{BI}=0.5 \mathrm{~s}$ shows outperformance. The neighbor timeout timer at $\alpha=2$ performs better for lower traffic loads and highly mobile and dense scenarios. On the other hand, in high network traffic and low mobility, $\alpha=3$ perform better than other settings. As future work, we plan to design an intelligent beaconing scheme that can adapt itself according to the mobility characteristics of UAVs.

\section{LIST OF ABBREVIATIONS}

The abbreviations used throughout the manuscript are listed below:

AODV Ad hoc on-demand distance vector

BI

Beacon interval

CBR

Constant bit rate

FANETS Flying ad hoc networks

GPSR Greedy perimeter stateless routing

MAC Media-access-control

MANETs Mobile ad hoc networks

$\mathrm{N}_{\text {TOT }}$

UAVs

Neighbor timeout timer

VANETs
Unmanned aerial vehicles

Vehicular ad hoc networks 


\section{REFERENCES}

[1] D. Shumeye Lakew, U. Sa'ad, N.-N. Dao, W. Na, and S. Cho, "Routing in Flying Ad Hoc Networks: A Comprehensive Survey," IEEE Commun. Surv. Tutorials, vol. 22, no. 2, pp. 1071-1120, 2020, doi: 10.1109/COMST.2020.2982452.

[2] A. Ashima, S. Krishna Pal, and Nonita, "A Comparative Analysis of Mobility Models for Network of UAVs," in In Proceedings of ICETIT 2019, Springer, Cham, 2020, pp. 36-47.

[3] O. S. Oubbati, M. Atiquzzaman, P. Lorenz, M. H. Tareque, and M. S. Hossain, "Routing in Flying Ad hoc Networks: Survey, Constraints and Future Challenge Perspectives," IEEE Access, vol. 7, pp. 1-1, 2019, doi: 10.1109/access.2019.2923840.

[4] J. Jiang and G. Han, "Routing Protocols for Unmanned Aerial Vehicles," IEEE Commun. Mag., vol. 56, no. 1, pp. 58-63, 2018, doi: 10.1109/MCOM.2017.1700326.

[5] A. Alkhatieb and E. Felemban, "Performance Evaluation of Ad Hoc Routing Protocols in (FANETs)," 2019 Int. Conf. Adv. Emerg. Comput. Technol. AECT 2019, 2020, doi: 10.1109/AECT47998.2020.9194201.

[6] O. S. Oubbati, A. Lakas, F. Zhou, M. Güneş, and M. B. Yagoubi, "A survey on position-based routing protocols for Flying Ad hoc Networks (FANETs)," Veh. Commun., vol. 10, pp. 29-56, 2017, doi: 10.1016/j.vehcom.2017.10.003.

[7] A. Bujari, C. E. Palazzi, and D. Ronzani, "A Comparison of Stateless Position-based Packet Routing Algorithms for FANETs," IEEE Trans. Mob. Comput., vol. 17, no. 11, pp. 2468-2482, 2018, doi: 10.1109/TMC.2018.2811490.

[8] S. M. Das, H. Pucha, and Y. Charlie Hu, "Performance comparison of scalable location services for geographic ad hoc routing," Proc. IEEE INFOCOM, vol. 2, no. C, pp. 1228-1239, 2005, doi: 10.1109/INFCOM.2005.1498349.

[9] B. Karp and H. T. Kung, "GPSR: Greedy Perimeter Stateless Routing for wireless networks," Proc. Annu. Int. Conf. Mob. Comput. Networking, MOBICOM, no. MobiCom, pp. 243-254, 2000.

[10] D. Son, A. Helmy, and B. Krishnamachari, "The effect of mobility-induced location errors on geographic routing in ad hoc networks: Analysis and improvement using mobility prediction," 2004 IEEE Wirel. Commun. Netw. Conf. WCNC 2004, vol. 1, no. 3, pp. 189-194, 2004, doi: 10.1109/wcnc.2004.1311541.
[11] R. Alsaqour et al., "Effect of mobility parameters on the inaccuracy of the position information of position-based MANET routing," Int. J. Wirel. Mob. Comput., vol. 7, no. 1, pp. 68-77, 2014, doi: 10.1504/IJWMC.2014.058886.

[12] M. Heissenbüttel, T. Braun, M. Wälchli, and T. Bernoulli, "Evaluating the limitations of and alternatives in beaconing," Ad Hoc Networks, vol. 5, no. 5, pp. 558-578, 2007, doi: 10.1016/j.adhoc.2006.03.002.

[13] N. Hernandez-Cons, S. Kasahara, and Y. Takahashi, "Dynamic hello/timeout timer adjustment in routing protocols for reducing overhead in MANETs," Comput. Commun., vol. 33 , no. 15 , pp. 1864-1878, 2010, doi: 10.1016/j.comcom.2010.06.011.

[14] R. Alsaqour et al., "Dynamic packet beaconing for GPSR mobile ad hoc position-based routing protocol using fuzzy logic," J. Netw. Comput. Appl., vol. 47, pp. 32-46, 2015, doi: 10.1016/j.jnca.2014.08.008.

[15] I. Benkhelifa, S. Moussaoui, and I. Demirkol, "Intertwined localization and error-resilient geographic routing for mobile wireless sensor networks," Wirel. Networks, vol. 26, no. 3, pp. 1731-1753, 2020, doi: 10.1007/s11276-018-18367.

[16] P. Neelagiri, V. M. Bhaskaran, and P. E. Linda, "Effective beaconing for better throughput in MANET," Int. J. Wirel. Mob. Comput., vol. 17, no. 1, pp. 43-53, 2019, doi: 10.1504/IJWMC.2019.101026.

[17] Z. S. Houssaini, I. Zaimi, M. Drissi, M. Oumsis, and S. E. A. Ouatik, "Trade-off between accuracy, cost, and QoS using a beacon-on-demand strategy and Kalman filtering over a VANET," Digit. Commun. Networks, vol. 4, no. 1, pp. 13-26, 2018, doi: 10.1016/j.dcan.2017.09.001.

[18] M. Naderi, F. Zargari, and M. Ghanbari, "Adaptive beacon broadcast in opportunistic routing for VANETs," Ad Hoc Networks, vol. 86, pp. 119130, 2019, doi: 10.1016/j.adhoc.2018.11.011.

[19] G. S. Rawat and K. Singh, "Joint beacon frequency and beacon transmission power adaptation for internet of vehicles," Trans. Emerg. Telecommun. Technol., no. July, pp. 1-15, 2020, doi: 10.1002/ett.4124.

[20] I. Mahmud and Y. Z. Cho, "Adaptive Hello Interval in FANET Routing Protocols for Green UAVs," IEEE Access, vol. 7, pp. 63004-63015, 2019, doi: 10.1109/ACCESS.2019.2917075. 
[21] X. Li and J. Huang, "ABPP: An adaptive beacon scheme for geographic routing in FANET," Parallel Distrib. Comput. Appl. Technol. PDCAT Proc., vol. 2017-Decem, pp. 293-299, 2018, doi: 10.1109/PDCAT.2017.00055.

[22] S. M. Daas, B. Zakaria, and S. Chikhi, "An Optimized Energy-Efficient Mission-Based Routing Protocol for Unmanned Aerial Vehicles," in International Symposium on Modelling and Implementation of Complex Systems, 2020, pp. 62-76.

[23] X. Li, F. Deng, and J. Yan, "Mobility-assisted adaptive routing for intermittently connected FANETs," IOP Conf. Ser. Mater. Sci. Eng., vol. 715 , no. 1, 2020, doi: 10.1088/1757$899 X / 715 / 1 / 012028$.

[24] M. F. Khan, K. L. A. Yau, R. M. Noor, and M. A. Imran, "Routing schemes in FANETs: A survey," Sensors (Switzerland), vol. 20, no. 1, pp. 1-33, 2020, doi: $10.3390 / \mathrm{s} 20010038$.

[25] Q. Chen, S. S. Kanhere, and M. Hassan, "Adaptive position update for geographic routing in mobile Ad Hoc networks," IEEE Trans. Mob. Comput., vol. 12, no. 3, pp. 489-501, 2013, doi: 10.1109/TMC.2012.20.

[26] K. Yu, L. Tan, L. Lin, X. Cheng, Z. Yi and T. Sato, "Deep-Learning-Empowered Breast Cancer Auxiliary Diagnosis for 5GB Remote E-Health," IEEE Wireless Communications, vol. 28, no. 3, pp. 54-61, June 2021, doi: 10.1109/MWC.001.2000374.

[27] K. Yu, L. Tan, S. Mumtaz, S. Al-Rubaye, A. AlDulaimi, A. K. Bashir, F. A. Khan, "Securing Critical Infrastructures: Deep Learning-based Threat Detection in the IIoT", IEEE Communications Magazine, 2021.

[28] K. Yu, Z. Guo, Y. Shen, W. Wang, J. C. Lin, T. Sato, "Secure Artificial Intelligence of Things for Implicit Group Recommendations", IEEE Internet of Things Journal, 2021, doi: 10.1109/JIOT.2021.3079574.

[29] L. Zhen, A. K. Bashir, K. Yu, Y. D. Al-Otaibi, C. H. Foh, and P. Xiao, "Energy-Efficient Random Access for LEO Satellite-Assisted 6G Internet of Remote Things", IEEE Internet of Things Journal, doi: 10.1109/JIOT.2020.3030856.

[30] Z. Guo, A. K. Bashir, K. Yu, J. C. Lin, Y. Shen, "Graph Embedding-based Intelligent Industrial Decision for Complex Sewage Treatment Processes", International Journal of Intelligent Systems, 2021, doi: 10.1002/int.22540.
[31] Z. Guo, K. Yu, A. Jolfaei, A. K. Bashir, A. O Almagrabi, and N. Kumar, "A Fuzzy Detection System for Rumors through Explainable Adaptive Learning”, IEEE Transactions on Fuzzy Systems, doi: 10.1109/TFUZZ.2021.3052109.

[32] Puttamadappa, C., and B. D. Parameshachari. "Demand side management of small scale loads in a smart grid using glow-worm swarm optimization technique." Microprocessors and Microsystems 71 (2019): 102886.

[33] Parameshachari, B. D., H. T. Panduranga, and Silvia liberata Ullo. "Analysis and computation of encryption technique to enhance security of medical images." In IOP Conference Series: Materials Science and Engineering, vol. 925, no. 1, p. 012028. IOP Publishing, 2020.

[34] Rajendran, Ganesh B., Uma M. Kumarasamy, Chiara Zarro, Parameshachari B. Divakarachari, and Silvia L. Ullo. "Land-use and land-cover classification using a human group-based particle swarm optimization algorithm with an LSTM Classifier on hybrid pre-processing remote-sensing images." Remote Sensing 12, no. 24 (2020): 4135.

[35] Seyhan, Kübra, Tu N. Nguyen, Sedat Akleylek, Korhan Cengiz, and SK Hafizul Islam. "Bi-GISIS KE: Modified key exchange protocol with reusable keys for IoT security." Journal of Information Security and Applications 58 (2021): 102788.

[36] Nguyen, Tu N., Bing-Hong Liu, Nam P. Nguyen, and Jung-Te Chou. "Cyber security of smart grid: attacks and defenses." In ICC 2020-2020 IEEE International Conference on Communications (ICC), pp. 1-6. IEEE, 2020.

[37] Naeem, Muhammad Ali, Tu N. Nguyen, Rashid Ali, Korhan Cengiz, Yahui Meng, and Tahir Khurshaid. "Hybrid Cache Management in IoTbased Named Data Networking." IEEE Internet of Things Journal (2021).

[38] Subramani, Prabu, K. Srinivas, R. Sujatha, and B. D. Parameshachari. "Prediction of muscular paralysis disease based on hybrid feature extraction with machine learning technique for COVID-19 and post-COVID-19 patients." Personal and Ubiquitous Computing (2021): 1-14.

[39] Bhuvaneswary, N., S. Prabu, K. Tamilselvan, and K. G. Parthiban. "Efficient Implementation of Multiply Accumulate Operation Unit Using an Interlaced Partition Multiplier." Journal of Computational and Theoretical Nanoscience 18, no. 4 (2021): 1321-1326. 